\title{
Origin of anomalous inverse notch effect in bulk metallic glasses
}

 \\ ${ }^{1}$ Shenyang National Laboratory for Materials Science, Institute of Metal Research, \\ Chinese Academy of Sciences, Shenyang 110016, China. \\ ${ }^{2}$ School of Engineering, Brown University, Providence, Rhode Island 02912, USA. \\ ${ }^{3}$ Centre for Advanced Mechanics and Materials, AML, Department of Engineering \\ Mechanics, Tsinghua University, Beijing 100084, China. \\ ${ }^{4}$ Department of Materials Science and Engineering, National University of Singapore, \\ 117576, Singapore.
}

${ }^{\mathrm{a} J . P . ~ a n d ~ H . Z . ~ c o n t r i b u t e d ~ e q u a l l y ~ t o ~ t h i s ~ w o r k . ~}$

*Corresponding authors: liyi@imr.ac.cn, huajian_gao@brown.edu. 


\begin{abstract}
Understanding notch-related failure is crucial for the design of reliable engineering structures. However, substantial controversies exist in the literature on the notch effect in bulk metallic glasses (BMGs), and the underlying physical mechanism responsible for the apparent confusion is still poorly understood. Here we investigate the physical origin of an inverse notch effect in a $\mathrm{Zr}$-based metallic glass, where the tensile strength of the material is dramatically enhanced, rather than decreased (as expected from the stress concentration point of view), by introduction of a notch. Our experiments and molecular dynamics simulations show that the seemingly anomalous inverse notch effect is in fact caused by a transition in failure mechanism from shear banding at the notch tip to cavitation and void coalescence. Based on our theoretical analysis, the transition occurs as the stress triaxiality in the notched sample exceeds a material-dependent threshold value. Our results fill the gap in the current understanding of BMG strength and failure mechanism by resolving the conflicts on notch effects and may inspire re-interpretation of previous reports on BMG fracture toughness where pre-existing notches were routinely adopted.
\end{abstract}

Keywords: Bulk metallic glasses, Notch, Strength, Shear band, Cavitation 


\section{Introduction}

Notch-related deformation and failure play a key role in the application of structural materials (Barenblatt, 1985; Bazant and Oh, 1983; Bazant, 1984; Bazant and Planas, 1998; Bilby et al., 1963; Dugdale, 1960; Huang et al., 2009; Suo et al., 1993; Tada et al., 1985). In small scale materials, it has been well documented that failure does not need to occur at stress concentrators as the characteristic size of the defects falls below a critical value (Bonderer et al., 2008; Gao et al., 2003; Gao et al., 2004; Gao and Yao, 2004; Giesa et al., 2012; Gu et al., 2013; Kumar et al., 2009; Kumar et al., 2011; Kumar et al., 2013; Qin and Buehler, 2011; Sha et al., 2013; Zhang et al., 2012). In bulk materials, however, failure is usually made easier by stress concentration around crack-like notches (Bilby et al., 1963; Dugdale, 1960; Tada et al., 1985). It is widely believed that the presence of notches leads to reduction in material strength.

Bulk metallic glasses (BMGs), a relatively new class of quasi-brittle materials, have attracted worldwide attention owing to their unique mechanical, physical and chemical properties (Ashby and Greer, 2006; Johnson, 1999). For example, the strength of ferrous BMGs can be as high as $5 \mathrm{GPa}$ (Inoue et al., 2003). To date, BMGs with superior fracture toughness up to $80 \mathrm{MPa} \cdot \mathrm{m}^{1 / 2}$ or even higher have been reported (Demetriou et al., 2011; He et al., 2012; Schroers and Johnson, 2004; Xu et al., 2010), making them potential materials for structural applications.

However, plastic deformation of BMGs under uniaxial tension tends to localize in a narrow region called shear band, leading to a self-focusing concentration of plastic strains and catastrophic failure of the material (Greer et al., 2013; Pan et al., 2011; 
Schuh et al., 2007). If an initial notch/crack is introduced, the resulting stress concentration would promote extensive shear banding near the tip region, giving rise to locally enhanced plasticity (Demetriou et al., 2011; Schroers and Johnson, 2004; Schuh et al., 2007). Considerable research has been devoted to understanding the effect of notches on the strength of BMGs. However, there remain substantial controversies in the literature. As expected by the notion of stress concentration, BMGs usually showed a notch-induced reduction in strength (Flores and Dauskardt, 2007; Henann and Anand, 2009; Varadarajan and Lewandowski, 2010), with the shear banding regarded as the root cause for fracture. Recently, it has been found that several BMGs in plate geometry exhibit notch insensitive strength, in that the tensile strength is barely affected by a notch (Sha et al., 2013; Qu et al., 2012; Qu et al., 2014). Possible enhancement in yielding stress due to the introduction of a notch is rarely reported (Kimura and Masumoto, 1983), and so far the reason for this inverse notch effect is still unclear. These apparently paradoxical or contradictory findings are calling for a careful investigation of notch-induced mechanical behaviors of BMGs.

The present work attempts to interpret the physical origin of inverse notch effect in a $\mathrm{Zr}$-based BMG subject to tensile test at room temperature. We will show that, as the stress triaxiality in front of the notch reaches a critical threshold value, the deformation mechanism would switch from a brittle failure mode governed by shear band formation to a ductile failure mode dominated by void nucleation, growth and coalescence, leading to a dramatic enhancement in strength. Such hitherto unknown transition in failure mechanism from shear banding to cavitation helps clarify the 
numerous conflicting reports on notch effect in BMGs over several decades (Flores and Dauskardt, 2007; Henann and Anand, 2009; Kimura and Masumoto, 1983; Sha et al., 2013; Qu et al., 2012; Qu et al., 2014; Varadarajan and Lewandowski, 2010).

\section{Methods}

\subsection{Experiments}

BMG with a nominal composition of $\mathrm{Zr}_{64.13} \mathrm{Cu}_{15.75} \mathrm{Ni}_{10.12} \mathrm{Al}_{10}$ (at. \%) was prepared by arc-melting mixtures of high purity metals (above 99.9\%) in a Ti-gettered high-purity argon atmosphere. Cylindrical samples with a diameter of $5 \mathrm{~mm}$ and a length of $75 \mathrm{~mm}$ were fabricated by tilt-casting into a copper mould. Notched tensile samples with notch dimensions (notch diameter $d \times$ notch height $h$ ) of $2.7 \times 0.45 \mathrm{~mm}^{2}$ (ND6) and $2.7 \times 2.7 \mathrm{~mm}^{2}$ (ND1) were produced by gently grinding in a custom-made machine, followed by fine polishing and final cleaning in an ultrasonic bath. The same procedure was adopted to prepare double-notched samples with notch dimensions of $2.7 \times 0.45 \mathrm{~mm}^{2}$. In such testing, catastrophic failure occurs at one of the notches. The details of plastic deformation right before fracture was then revealed on the cross section of the surviving notch.

Quasi-static tensile tests were carried out at room temperature using an electro-servo-hydraulic system (Instron 8521 machine) with a maximum load of 100 $\mathrm{KN}$ at a cross head speed of $0.02 \mathrm{~mm} / \mathrm{min}$, corresponding to a constant engineering

strain rate of $5 \times 10^{-4} / \mathrm{s}$ in the notched region. An extensometer was attached across the notch to calibrate and measure the elongation during the test. Fracture morphology 
was examined by SEM (Philips XL30 FEG instrument).

\subsection{Simulations}

Molecular dynamics (MD) simulations were performed on two notched cylindrical pillars of $\mathrm{CuZr}$ glass containing a total of about 10,000,000 atoms, as shown in Fig. 1(a) and 1(b). The notch diameter $d$ was chosen as $30 \mathrm{~nm}$ for both samples to prevent necking upon yielding. The notch height $h$ was taken to be $30 \mathrm{~nm}$ in ND1 and $5 \mathrm{~nm}$ in ND6. The height and diameter of the pillars were both $50 \mathrm{~nm}$. An embedded atom method potential for CuZr was adopted (Mendelev et al., 2007). The simulation time step was taken to be $1 \mathrm{fs}$. The glass structure was generated by a melt-quench method. A cubic cell (10,976 atoms) containing 64 at.\% Copper and 36 at.\% Zirconium was equilibrated at $2500 \mathrm{~K}$ and zero pressure for 200 ps to ensure homogeneity (Nose et al., 1984). A quenching rate of 50K/ns was used to cool the molten system from $2500 \mathrm{~K}$ to $1 \mathrm{~K}$. The final dimensions of the cubic cell were measured to be $5.6 \times 5.6 \times 5.6 \mathrm{~nm}^{3}$. The cylindrical pillars were constructed from the cubic cell replicas. The as-built pillars were equilibrated for 200 ps before straining. During uniaxial tension, periodic boundary condition was imposed in the axial direction, while the pillar surface in the radial direction is kept free. A constant strain rate of $1 \times 10^{8} \mathrm{~s}^{-1}$ was applied along the axial direction until a maximum of $20 \%$ strain was reached. To visualize plastic shearing during deformation, the local shear invariant $\eta^{\text {Mises }}$ of each atom in the sample was calculated using (Shimizu et al., 2007)

$$
\eta^{\text {Mises }}=\sqrt{\frac{1}{2} \operatorname{Tr}\left\{\left(\eta-\eta_{m} \mathrm{I}\right)^{2}\right\}}
$$

where $\eta$ and $\eta_{m}$ are local Lagrangian strain and local hydrostatic strain associated 
with the atom, respectively. Atoms with a value of $\eta^{\text {Mises }}$ larger than 0.03 were regarded as atoms in an active shear transformation zone (STZ).

The symmetry of the notched specimens allowed us to use axisymmetric quadrilateral elements in finite element modeling (FEM), as shown in Fig. 1(c) and 1(d). Appropriate symmetry conditions were imposed on the boundaries $X_{1}=X_{2}=0$. The system was loaded by applying a uniform displacement of $u$ on the boundary $X_{2}=L$, giving a macroscopic logarithmic strain of $\ln (1+u / L)$ along the $X_{2}$ direction. The stress-strain relation of the material was assumed to obey the elastic perfectly plastic law:

$$
\begin{aligned}
& \sigma=E \varepsilon \text { if }|\varepsilon| \leq \varepsilon_{y} \\
& \sigma=\sigma_{y} \operatorname{sign}(\varepsilon) \text { if }|\varepsilon|>\varepsilon_{y}
\end{aligned}
$$

where $E, \sigma_{\mathrm{y}}$ and $\varepsilon_{\mathrm{y}}$ represent the Young's modulus, yield stress and strain, respectively. The function $\operatorname{sign}(\varepsilon)$ was defined as follows:

$$
\operatorname{sign}(\varepsilon)=\left\{\begin{array}{ccc}
1 & \text { if } & \varepsilon>0 \\
0 & \text { if } & \varepsilon=0 \\
-1 & \text { if } & \varepsilon<0
\end{array}\right.
$$

In our simulations, the Young's modulus $E$, yield strength $\sigma_{\mathrm{y}}$ and the Poisson's ratio $v$ of $\mathrm{CuZr}$ were taken as $90 \mathrm{GPa}, 1.6 \mathrm{GPa}$ and 0.35 , respectively. Pressure contours were extracted from the simulations at a logarithmic strain of $8 \%$ to evaluate the triaxial stress state in the notched samples.

\section{Results and Discussion}

Fig. 2(a) shows the stress-strain curves of the pre-notched specimens ND1 (a shallow-notched sample with $d / h=1$ ) and ND6 (a deep-notched sample with $d / h=6$ ). 
The insets show scanning electron microscopy (SEM) images of the specimens in the unloaded condition. It can be seen that ND1 reaches a peak stress of $1.6 \mathrm{GPa}$ at about $2 \%$ elastic strain and then fails with negligible plasticity, corresponding to a typical brittle failure behavior. For the deep-notched sample ND6, the specimen surprisingly did not fail at a smaller peak stress in a similarly brittle manner, as would be expected from the conventional wisdom about the effect of stress concentration at the notch tip [e.g., Pilkey et al. (2007) gives a stress concentration factor of about 2.5 for ND6]. Rather, the peak stress of ND6 reaches an ultrahigh value of $3.3 \mathrm{GPa}$, with a substantial portion of the stress-strain curve exhibiting a nonlinear, strain hardening behavior prior to final failure (Wang et al., 2013). To better illustrate this ultra-strength, we summarize the relationship between fracture strength and Young's modulus for both amorphous and crystalline metals in Fig. 2(b). It is seen that amorphous alloys commonly possess an average fracture strength about $2 \%$ of their Young's modulus, which is higher than those reported for crystalline metals $(\sim 0.65 \%)$ (Inoue et al., 2006; Yuan and Xi, 2011). In comparison, the fracture strength of ND6 is approaching the theoretical limit $(E / 30-E / 10)$ of the material.

To understand the physical origin of the observed inverse notch effect, we studied the deformation and fracture process by examining the fracture surface morphology of the two specimens, as shown in Fig. 3. It is seen that ND1 fractures at an angle of about $50^{\circ}$ with respect to the loading axis [Fig. 3(a)]. A radial vein-like pattern with a length scale of $20-50 \mu \mathrm{m}$ was observed on the flat fracture surface, as revealed by the post-mortem SEM image shown in Fig. 3(b). The failure plane of ND6 is, however, 
perpendicular to the loading axis, as shown in Fig. 3(c). Closer examination of the fracture surface showed that the sample interior is filled with profuse dimples and micro-cracks in an uneven ridge-like pattern [see the inset of Fig. 3(d)]. The average size of the dimples is $10-20 \mu \mathrm{m}$, much larger than the nanoscale dimple-like structures observed on the tensile fracture surface of brittle BMGs (Xi et al., 2005). Shear traces were observed outside the dimple zone, indicating direct link-ups between the inner cracks and the notch surface when catastrophic failure occurs. To better understand the details of deformation right prior to the final catastrophic failure, we performed an additional tensile test on a double-notched specimen (Lewandowski and Greer, 2006), as shown in Fig. 3(e), with starter notches having the same dimensions as that in ND6. The double-notched specimen allowed us to capture the near critical state of deformation in the surviving notch after the other notch fails. Numerous micro-cracks with length scales of 10-100 $\mu \mathrm{m}$ were observed in the cross section. Figure 3(f) shows an enlarged SEM image of a particular crack located at the center of the surviving notch region. It was found that the crack plane is highly serrated, and the dimension of the constituent crack segments is on the order of $0.2-2 \mu \mathrm{m}$. It was also seen that the angle between the crack plane and loading direction is roughly $90^{\circ}$, which is completely different from that associated with conventional shear banding.

The dimples and micro-cracks observed on the mode I fracture surface in the deep-notched sample ND6 suggest nucleation, growth and coalescence of voids during deformation (Huang et al., 1991; McClintock, 1968; Rice and Tracey, 1969). To complement the experimental results, we performed large-scale MD simulations to 
investigate the notch effects at the atomic scale. We considered a simpler binary $\mathrm{CuZr}$ MG as there is a current lack of appropriate interatomic potential for the alloy used in our experiments. Despite the simplified model, the simulation results show similar behaviors to our experimental observations. The simulated samples, also referred to as ND1 and ND6, were directly scaled down from the experiments. Note that the notch diameter $d$ is carefully chosen in order to reproduce shear banding failure when the sample is notch-free. Fig. 4(a) displays the simulated stress versus strain curves of the two pre-notched samples, where the tensile stress has been normalized by the cross sectional area of the starter notch. It can be seen that the peak stress of ND6 is approximately 1.27 times larger than that of ND1, in qualitative agreement with our experiments. The quantitative difference between simulations and experiments can be attributed to the fact that, due to its nanoscale size, the sample ND1 in MD simulations already has a strength closer to the theoretical limit, so there is less room for further enhancement compared to that in experiments.

Typical deformation patterns of the simulated samples at $15 \%$ strain are shown in Fig. 4(b) and 4(c). It is seen that plastic deformation in ND1 takes place along an inclined band-shaped region characteristic of a dominant shear band. The width of the shear band is approximately $10 \mathrm{~nm}$, in agreement with previous reports (Zhang and Greer, 2006). On the other hand, deformation in ND6 is controlled by void nucleation and growth at the center of the notched sample. Careful examination of the deformation process revealed that a nanoscale void is formed spontaneously at about 7.5\% strain, a process commonly referred to as cavitation. In this way, highly 
localized shear banding has been replaced by nucleation and growth of voids whose coalescence leads to mode I fracture. It should be mentioned that our simulation did not capture void coalescence owing to the limited sample size.

In view of the above analysis, the physical origin of the seemingly paradoxical inverse notch effect is revealed as a transition from shear banding governed catastrophic failure to void nucleation, growth and coalescence in front of the notch. To develop a theoretical model to rationalize this notch-induced transition in failure mechanism, we first took a look at the stress distribution in the notched specimens. We conducted FEM simulations of the notched BMG specimens under tension. Contours depicting triaxial stress distribution in the notched samples at $8 \%$ of tensile strain are shown in Fig. 5(a) and 5(b). Compared to the otherwise uniform distribution in notch-free samples, the triaxial stress in the two notched specimens exhibits a very different pattern. It is seen that the maximum triaxial stress (equivalent to the minimum pressure) occurs at the center of the notch. The intensity of stress concentration is sensitive to the dimension of the starter notch. The maximum triaxial stress of ND6 is roughly 3 times larger than that of ND1. The nucleation, growth and coalescence of voids caused by the strong traixial stress field provide an explanation for the observed deep dimples on the fracture surface in ND6. Similar growth of internal voids in highly constrained metal wires under tension was previously observed by Ashby et al. (1989).

Based on the above insights, one may understand the transition in failure mechanism by considering the level of stress triaxiality at the center of the notched section of the sample (Bridgman, 1964): 


$$
\frac{\sigma_{\mathrm{m}}}{\sigma_{\mathrm{eq}}}=\frac{1}{3}+\ln \left(1+\frac{d}{2 h}\right)
$$

where $\sigma_{\mathrm{m}}$ is the hydrostatic stress and $\sigma_{\mathrm{eq}}$ the von Mises equivalent stress, respectively. Eq. (1) provides a quantitative description of the competition between void formation and shear banding. For the notch-free samples, the logarithmic term in Eq. (1) equals to zero. In this circumstance, shear banding acts as the dominating deformation and failure mechanism. In the notched samples, $\sigma_{\mathrm{m}} / \sigma_{\mathrm{eq}}$ increases with $d / h$. As $\sigma_{\mathrm{m}} / \sigma_{\mathrm{eq}}$ reaches a threshold value (Fig. 6) defined by $\sigma_{\mathrm{c}} / \sigma_{\mathrm{s}}$ where $\sigma_{\mathrm{c}}$ and $\sigma_{\mathrm{s}}$ denote the critical stresses for cavitation and shear localization, respectively, the failure mode would switch from shear banding to nucleation and growth of voids whose coalescence leads to mode I fracture [Fig. 3(c)]. Clearly, $\sigma_{\mathrm{c}} / \sigma_{\mathrm{s}}$ can be a material-dependent parameter. To estimate $\sigma_{\mathrm{c}} / \sigma_{\mathrm{s}}$, we performed additional traixial and uniaxial tensile simulations on a sample with dimensions $20 \times 20 \times 20 \mathrm{~nm}^{3}$. The calculated value of $\sigma_{\mathrm{c}} / \sigma_{\mathrm{s}}$ is $\sim 2.6$. It has been recently demonstrated that the atomic scale fluctuation of local mechanical properties could play important roles in the fracture behavior of amorphous alloys (Guan et al., 2013; Murali et al., 2011; Wagner et al., 2011). Therefore, we carried out a series of triaxial tensile simulations on samples with extremely small dimensions (comparable to the cutoff distance of the potential). The fluctuation was estimated to be on the order of $\sim 10 \%$. Since casting defects such as voids are quite common in amorphous alloys (Lee et al., 2010), we also conducted a series of triaxial tensile simulations on samples with an initial void content (measured by its volume fraction) ranging from $0 \%$ to $3.35 \%$. It was found that a void content on the order of $1 \%$ could lead to a reduction in cavitation stress on the order of $\sim 40 \%$. Taking the above factors 
into account, we calculated the critical stress triaxiality $\sigma_{\mathrm{c}} / \sigma_{\mathrm{s}}$ to be $\sim 1.4$. Based on Eq. (1), sample ND1 has a stress triaxiality of 0.74 which is lower than $\sigma_{\mathrm{c}} / \sigma_{\mathrm{s}}$, hence expected to fail by shear localization, as shown in Fig. 3(a) and 3(b). On the other hand, the stress triaxiality of sample ND6 is 1.72 , larger than the threshold value set by $\sigma_{\mathrm{c}} / \sigma_{\mathrm{s}}$, which explains the dimple associated mode I fracture shown in Fig. 3(c) and $3(d)$.

BMGs usually exhibit quasi-brittle tensile behavior due to shear banding induced catastrophic failure (Greer et al., 2013; Pan et al., 2011; Schuh et al., 2007). The deep notch in ND6 leads to a much reduced effective shear stress in front of it (Brown et al., 1954; Flores and Dauskardt, 2001), effectively hindering the activation and propagation of shear band. In this state, the material is able to continuously deform (see Supplementary Movie recording the large elongation of the deep notch in ND6 before fracture) until spontaneous void nucleation occurs at a sufficiently high triaxial stress state, possibly with the aid of pre-existing casting defects. Upon further straining, the continuous growth and coalesce of voids lead to mode I cracks, as evidenced by the observed dimples and micro-cracks on the fracture surface [the inset of Fig. 3(c) and 3(d)]. Once reaching the critical size, the micro-cracks would link up with the surface and shear off. The observed micro-cracks are highly serrated and may interact with each other as the deformation proceeds along the nonlinear stress-strain curve shown in Fig. 2(a) (Gludovatza et al., 2013). Our theoretical model suggests that a change in the chemical composition of BMG corresponds to a change of the threshold value $\sigma_{\mathrm{c}} / \sigma_{\mathrm{s}}$, and thus may require a different threshold value of stress 
triaxiality for the brittle-to-ductile transition. We could thus speculate that previous studies in the literature did not cover a sufficient level of stress triaxiality and therefore missed the inverse notch effect and the associated transition in failure mechanism in BMGs (Flores and Dauskardt, 2007; Henann and Anand, 2009; Qu et al., 2012; Qu et al., 2014; Varadarajan and Lewandowski, 2010).

Fig. 7 shows representative experimental data summarizing different types of notch effect in metallic glasses reported in the literature, including positive notch effect in cylindrical samples (Flores and Dauskardt, 2007), notch insensitivity in notched plate samples (Qu et al., 2012; Qu et al., 2014) and inverse notch effect in cylindrical samples (Kimura and Masumoto, 1983). For comparison, we also plot our current data, including ND1, ND6 and two additional notched specimens with intermediate notch dimensions, ND2.3 and ND4, as well as the notch-free case ND0. Similar behaviors have also been observed in other materials systems (Farbaniec et al., 2013; Kondori and Benzerga, 2014; Pineau et al., 2006; Sobieraj et al., 2005).

\section{Conclusions}

We have provided an explanation for the inverse notch effect in a Zr-based BMG by relating the strength increase to a transition in failure mechanism from shear banding to cavitation caused by a sufficiently high triaxial stress state. Our results help explain the seemingly conflicting results in the literature on notch effects in BMGs. It should be pointed out that a simple integration of the stress-strain curves [Fig. 2(a)] implies that there is a 15 fold increase in the energy absorption capability 
of ND6 before fracture compared to that of ND1, suggesting that appropriate design of macrostructure may considerably enhance the capability of BMGs to withstand potential impact or shock loading in service (Sarac and Schroers, 2013; Sarac et al., 2014). Therefore, the present work suggests that it may be necessary to reinterpret or re-examine some of the previous results on fracture toughness in monolithic BMGs, as pre-existing notches are widely adopted in fracture toughness tests (Demetriou et al., 2011; He et al., 2012; Schroers and Johnson, 2004; Xu et al., 2010). Based on our analysis, a deep notch might trigger cavitation and void coalescence far away from the notch in some BMGs, while the same notch might promote shear banding around the tip of the notch in other BMGs. Therefore, the fracture toughness data measured from different fracture testing techniques on various BMG geometries should be examined with care before comparison.

\section{Acknowledgements}

J. Pan and Y. Li acknowledge financial support by National Nature Science Foundation of China under Grant No.51401220 and 51471165. H. Zhou and H. Gao acknowledge financial support from the National Science Foundation through grant CMMI-1161749 and the Center of Mechanics and Materials at Tsinghua University. 


\section{References}

Ashby, M.F., Blunt, F.J., Bannister, M., 1989. Flow characteristics of highly constrained metal wires. Acta. Mater. 37, 1847-1857.

Ashby, M.F., Greer, A.L., 2006. Metallic glasses as structural materials. Scr. Mater. 54, 321-326.

Barenblatt, G.I., 1985. The mathematical theory of equilibrium cracks in brittle fracture. Adv. Appl. Mech., VII (Academic Press, New York), pp 69-89.

Bazant, Z.P., Oh, B.H., 1983. Crack band theory for fracture of concrete. Matériaux et Construction 16, 155-177.

Bazant, Z.P., 1984. Size effect in blunt fracture: concrete, rock, metal. J. Eng. Mech. $110,518-535$

Bazant, Z.P., Planas, J., 1998. Fracture and Size Effect in Concrete and Other Quasibrittle Materials (CRC Press, Boca Raton, FL), pp 1-112.

Bilby, B.A., Cottrell, A.H., Swinden, K.H., 1963. The spread of plastic yield from a notch. Proc. R. Soc. London, Ser. A 272, 304-314.

Bonderer, L.J., Studart, A.R., Gauckler, L.J., 2008. Bioinspired design and assembly of platelet reinforced polymer films. Science 319, 1069-1073.

Bridgman, P.W., 1964. Studies in large plastic flow and fracture. Harvard University Press, Cambridge, MA.

Brown, A.F.C., 1954. Triaxial stresses caused by notches. Br. J. Appl. Phys. 5, 280-284.

Demetriou, M.D., Launey, M.E., Garrett, G., Schramm, J.P., Hofmann, D.C., Johnson, 
W.L., Ritchie, R.O., 2011. A damage-tolerant glass. Nat. Mater. 10, 123-128.

Dugdale, D.S., 1960. Yielding of steel sheets containing slits. J. Mech. Phys. Solids 8, $100-104$.

Farbaniec, L., Couque, H., Dirras, G., 2013. Influence of triaxial stress state on ductile fracture strength of polycrystalline nickel. Int. J. Fract. 182, 267-274.

Flores, K.M., Dauskardt, R.H., 2001. Mean stress effects on flow localization and failure in a bulk metallic glass. Acta Mater. 49, 2527-2537.

Gao, H.J., Ji, B.H., Lager, I.L., Arzt, E., Fratzl, P., 2003. Materials become insensitive to flaws at nanoscale: Lessons from nature. Proc. Natl. Acad. Sci. USA 100, $5597-5600$.

Gao, H., Ji, B., Buehler, M. J., Yao, H., 2004. Flaw tolerant bulk and surface nanostructures of biological systems. Mech. Chem. Biosyst. 1, 37-52.

Gao, H., Yao, H., 2004. Shape insensitive optimal adhesion of nanoscale fibrillar structures. Proc. Natl. Acad. Sci. USA 101, 7851-7856.

Giesa, T., Pugno, N.M., Buehler, M.J. 2012. Natural stiffening increases flaw tolerance of biological fibers. Phys. Rev. E 86, 041902.

Gludovatza, B., Demetriou, M.D., Floyd, M., Hohenwarter, A., Johnson, W.L., Ritchie, R.O., 2013. Enhanced fatigue endurance of metallic glasses through a staircase-like fracture mechanism. Proc. Natl. Acad. Sci. USA 110, $18419-18424$.

Greer, A.L., Cheng, Y.Q., Ma, E., 2013. Shear bands in metallic glasses. Mater. Sci. Eng. R 74, 71-132. 
Gu, X.W., Wu, Z.X., Zhang, Y.W., Srolovitz, D.J., Greer, J.R., 2013. Microstructure versus flow: mechanisms of failure and strength in nanostructures. Nano Lett. 13, 5703-5709.

Guan, P., Lu, S., Spector, M.J.B., Valavala, P.K., Falk, M.L., 2013. Cavitation in amorphous solids. Phys. Rev. Lett. 110, 185502.

He, Q., Shan, J.K., Ma, E., Xu, J., 2012. Crack-resistance curve of a Zr-Ti-Cu-Al bulk metallic glass with extraordinary fracture toughness. Acta Mater. 60, 4940-4949.

Henann, D.L., Anand, L., 2009. Surface tension-driven shape-recovery of micro/nanometer-scale surface features in a Pt57.5Ni5.3Cu14.7P22.5 metallic glass in the supercooled liquid region: A numerical modeling capability. Acta Mater. 57, 6057.

Huang Y.G, Hutchinson JW, Tvergaard V (1991) Cavitation instabilities in elastic-plastic solids. J. Mechan. Phys. Solids 39(2): 223-241.

Huang, S., Zhang, S., Belytschko, T., Terdalkar, S.S., Zhu, T., 2009. Mechanics of nanocrack: fracture, dislocation emission, and amorphization. J. Mech. Phys. Solids 57, 840-850.

Inoue, A., Shen, B.L., Koshiba, H., Kato, H., Yavari, A.R., 2003. Cobalt-based bulk glassy alloy with ultrahigh strength and soft magnetic properties. Nat. Mater. 11, 661-663.

Inoue, A., Shen, B.L., Takeuchi, A., 2006. Developments and applications of bulk glassy alloys in late transition metal base system. Mater. Trans. 47, 1275-1285. 
Johnson, W.L., 1999. Bulk glass-forming metallic alloys: Science and technology. MRS Bull. 24, 42-56.

Kimura, H., Masumoto, T., 1983. Plastic constraint and ductility in tensile notched specimens of amorphous Pd78Cu6Si16. Metall. Trans. A 14, 709-716.

Kondori, B., Benzerga, A.A., 2014. Effect of stress triaxiality on the flow and fracture of Mg alloy AZ31. Metall. Mater. Trans. A 45, 3292-3307.

Kumar, S., Haque, M.A., Gao, H.J., 2009. Notch insensitive fracture in nanoscale thin films. Appl. Phys. Lett. 94, 253104.

Kumar, S., Li, X.Y., Haque, A., Gao, H.J., 2011. Is stress concentration relevant for nanocrystalline metals? Nano Lett. 11, 2510-2516.

Kumar, S., Haque, M.A., Gao, H.J., 2013. Transformation induced toughening and flaw tolerance in pure nanocrystalline aluminum. Int. J. Plasticity 44, 121-128.

Lee, C.J., Lai, Y.H., Huang, J.C., Du, X.H., Wang, L., Nieh, T.G., 2010. Strength variation and cast defect distribution in metallic glasses. Scr. Mater. 63, 105-108.

Lewandowski, J.J., Greer, A.L., 2006. Temperature rise at shear bands in metallic glasses. Nat. Mater. 5, 15-18.

McClintock, F.A., 1968. A criterion for ductile fracture by the growth of holes. J. Appl. Mechan. 35, 363-371.

Mendelev, M.I., Rehbein, D.K., Ott, R.T., Kramer, M.J., Sordelet, D.J., 2007. Computer simulation and experimental study of elastic properties of amorphous Cu-Zr alloys. J. Appl. Phys. 102, 093518.

Murali, P., Guo, T.F., Zhang, Y.W., Narasimhan, R., Li, Y., Gao, H.J., 2011. Atomic 
scale fluctuations govern brittle fracture and cavitation behavior in metallic glasses. Phys. Rev. Lett. 107, 215501.

Nose, S., 1984. A unified formulation of the constant temperature molecular dynamics methods. J. Chem. Phys. 81, 511-519.

Pan, J., Chen, Q., Liu, L., Li, Y., 2011. Softening and dilatation in a single shear band. Acta Mater. 59, 5146-5158.

Pilkey, W.D., Pikey, D.F., 2007. Peterson's Stress Concentration Factors 3rd ed. (John Wiley \& Sons Inc, Hoboken).

Pineau, A., 2006. Development of the local approach to fracture over the past 25 years: theory and applications. Int. J. Fract. 138, 139-166.

Qin, Z., Buehler, M.J., 2011. Flaw tolerance of nuclear intermediate filament lamina under extreme mechanical deformation. ACS Nano 5, 3034-3042.

Qu, R.T., Calin, M., Eckert, J., Zhang, Z.F., 2012. Metallic glasses: Notch-insensitive materials. Scr. Mater. 66, 733-736.

Qu, R.T., Zhang, P., Zhang, Z.F., 2014. Notch effect of materials: strengthening or weakening? J. Mater. Sci. Technol. 30, 599-608.

Rice, J.R., Tracey, D.M., 1969. On the ductile enlargement of voids in triaxial stress fields. J. Mechan. Phys. Solids 17, 201-217.

Sarac, B., Schroers, J., 2013. Designing tensile ductility in metallic glasses. Nat. Commun. 4, 2158.

Sarac, B., Klusemann, B., Xiao, T., Bargmann, S., 2014. Materials by design: An experimental and computational investigation on the microanatomy arrangement 
of porous metallic glasses. Acta Mater. 77, 411-422.

Schroers, J., Johnson, W.L., 2004. Ductile bulk metallic glass. Phys. Rev. Lett. 93, 255506.

Schuh, C.A., Hufnagel, T.C., Ramamurty, U., 2007. Mechanical behavior of amorphous alloys. Acta Mater. 55, 4067-4109.

Sha, Z.D., Pei, Q.X., Sorkin, V., Branicio, P.S., Zhang, Y.W., Gao, H.J., 2013. On the notch sensitivity of CuZr metallic glasses. Appl. Phys. Lett. 103, 081903.

Shimizu, F., Ogata, S., Li, J., 2007. Theory of shear banding in metallic glasses and molecular dynamics calculations. Mater. Trans. 48, 2923-2927.

Sobieraj, M.C., Kurtz, S.M., Rimnac, C.M., 2005. Notch strengthening and hardening behavior of conventional and highly crosslinked UHMWPE under applied tensile loading. Biomaterials 26, 3411-3426.

Suo, Z., Ho, S., Gong, X., 1993. Notch ductile-to-brittle transition due to localized inelastic band. J. Eng. Mater. Technol. 115, 319-326.

Tada, H., Paris, P.C., Irwin, G.R., 1985. The Stress Analysis of Cracks Handbook, 2nd ed. (Del Research Corporation, St. Louis), pp 1-52.

Varadarajan, R., Lewandowski, J.J., 2010. Stress-state effects on the fracture of a Zr-Ti-Ni-Cu-Be bulk amorphous alloy. Metall. Mater. Trans. A 41A, 1758-1766.

Wagner, H., Bedorf, D., Kuchemann, S., Schwabe, M., Zhang, B., Arnold, W., Samwer, K., 2011. Local elastic properties of a metallic glass. Nat. Mater. 10, 439-442.

Wang, Z.T., Pan, J., Li, Y., Schuh, C.A., 2013. Densification and strain hardening of a 
metallic glass under tension at room temperature. Phys. Rev. Lett. 111, 135504.

Xi, X.K., Zhao, D.Q., Pan, M.X., Wang, W.H., Wu, Y., Lewandowski, J.J., 2005. Fracture of brittle metallic glasses: brittleness or plasticity. Phys. Rev. Lett. 94, 125510.

Xu, J., Ramamurty, U., Ma, E., 2010. The fracture toughness of bulk metallic glasses. JOM 62, 10-18.

Yuan, C.C., Xi, X.K., 2011. On the correlation of Young's modulus and the fracture strength of metallic glasses. J. Appl. Phys. 109, 033515.

Zhang, T., Li, X.Y., Kadkhodaei, S., Gao, H.J., 2012. Flaw insensitive fracture in nanocrystalline graphene. Nano Lett. 12, 4605-4610.

Zhang, Y., Greer, A.L., 2006. Thickness of shear bands in metallic glasses. Appl. Phys. Lett. 89, 071907. 


\section{Figure Legends}

Fig. 1. Initial setups of MD (a) and FEM (b) simulations for the pre-notched metallic glasses ND1 and ND6.

Fig. 2. Inverse notch effect in Zr-based bulk metallic glasses. (a) Typical tensile stress versus strain relations of two notched specimens with different notch dimensions. The minimum load bearing area of the specimens was used to determine true stress from the raw load-displacement curves. Contrary to the conventional notion of stress concentration at the notch tip reducing the strength of materials, the deep notched sample ND6 exhibits much higher strength than the shallow notched sample ND1. The inset shows the typical SEM images of the specimens under tensile testing (scale bar, $1 \mathrm{~mm})$. (b) Fracture strength versus Young's modulus for typical amorphous and crystalline alloys.

Fig. 3. Fracture surface morphologies of the notched Zr-based bulk metallic glasses. (a) SEM image showing the typical shear fracture in ND1 at an angle of about $50^{\circ}$ with respect to the loading axis. (b) SEM image showing the radial vein-like pattern on the fracture surface of ND1, indicative of shear banding failure. (c) SEM image showing the mode I fracture in ND6 at $90^{\circ}$ with respect to the loading axis. (d) SEM image showing the rough fracture surface with profuse dimples and micro-cracks. $(e)$ Optical image of a double notched BMG specimen. $(f)$ A micro-crack formed at the center of the surviving notch after the sample fails at the other notch. The inset indicates the location of this micro-crack.

Fig. 4. Molecular dynamics simulations confirming the inverse notch effect in 
metallic glasses. (a) Simulated stress-strain curves of two notched samples with different notch dimensions. The calculated tensile stresses are normalized by the cross sectional area of the starter notch. The peak stress of ND6 is 1.25 times that of specimen ND1. The insets show the initial configurations of the samples (scale bars, $10 \mathrm{~nm}) .(b)-(c)$ Cross sectional view of the simulated deformation patterns of the notched MG pillars at $15 \%$ strain. (scale bars, $5 \mathrm{~nm}$.) In (b), plastic deformation localizes into an inclined shear band, whereas in $(c)$, a void appears near the center of the specimen as it fails by mode I fracture.

Fig. 5. Cross-sectional contours showing the distribution of pressure in the simulated samples at $8 \%$ strain. Varying the starter notch dimension from $d / h=1 / 1$ in ND1 $(a)$ to 6/1 in ND6 (b) almost triples the level of tensile triaxial stress at the center of the notch.

Fig. 6. Inverse notch effect governed by a transition from shear banding to void growth in bulk metallic glasses. A transition in deformation mechanism controlled by the ratio of stress triaxiality, $\sigma_{\mathrm{m}} / \sigma_{\mathrm{eq}}$, as a function of the notch dimension, $d / h$. The stress triaxiality increases with increasing notch dimension. Before reaching the critical value defined by $\sigma_{\mathrm{c}} / \sigma_{\mathrm{s}}$ (indicated by the horizontal red line), plastic deformation tends to localize in a dominant shear band. The pink window represents the uncertainty due to atomic scale fluctuations. When the stress triaxiality is larger than the critical value, material fails by void growth and coalescence. Note that $d / h$ equals to zero in a notch-free sample.

Fig. 7. Representative experimental data illustrating the different types of notch effect 
in metallic glasses, including the positive notch effect in cylindrical samples (Flores and Dauskardt, 2007), notch insensitivity in plate samples (Qu et al., 2012; Qu et al., 2014) and inverse notch effect observed previously (Kimura and Masumoto, 1983) and in the present study. Here, the normalized fracture strength is defined as the ratio of the fracture strength of the notched sample to that of the sample without notch. 
Figure 1

(a)

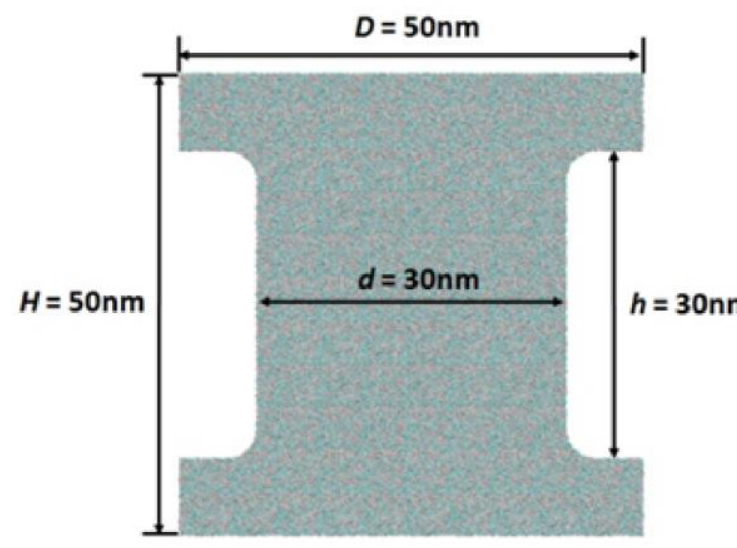

(c)

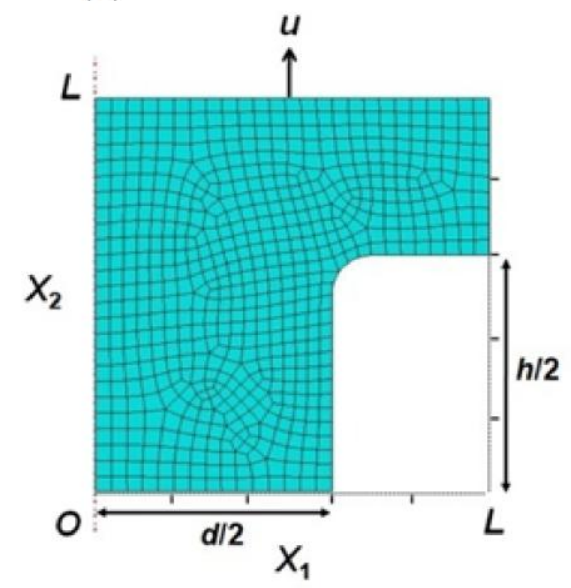

(b)



(d)

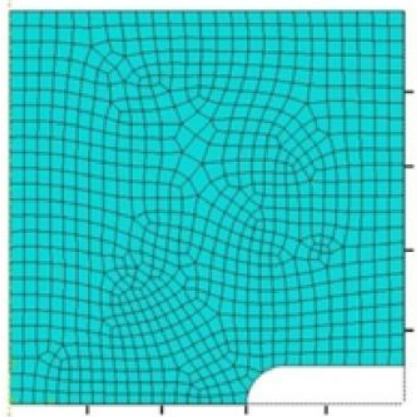


Figure 2
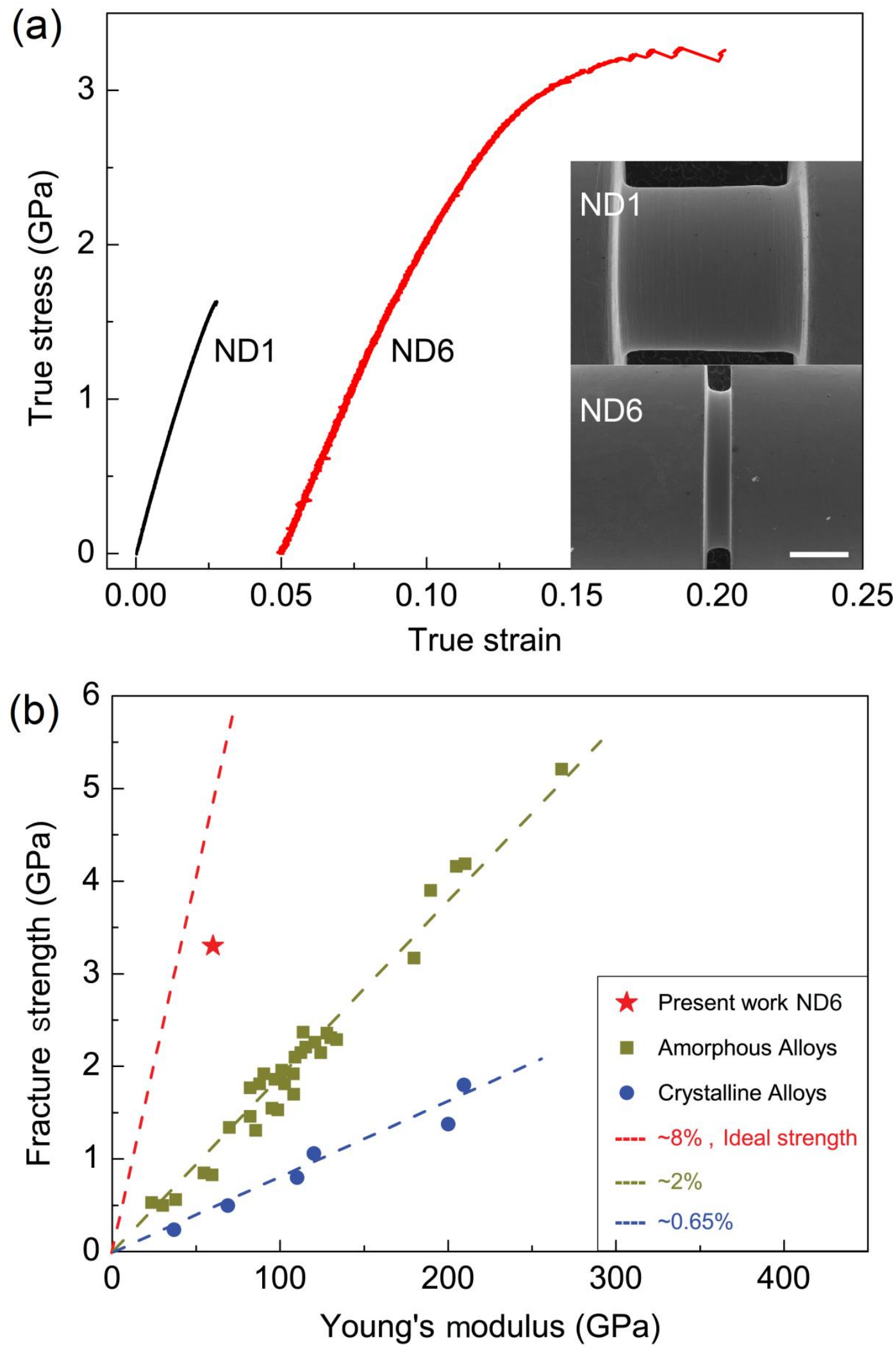
Figure 3

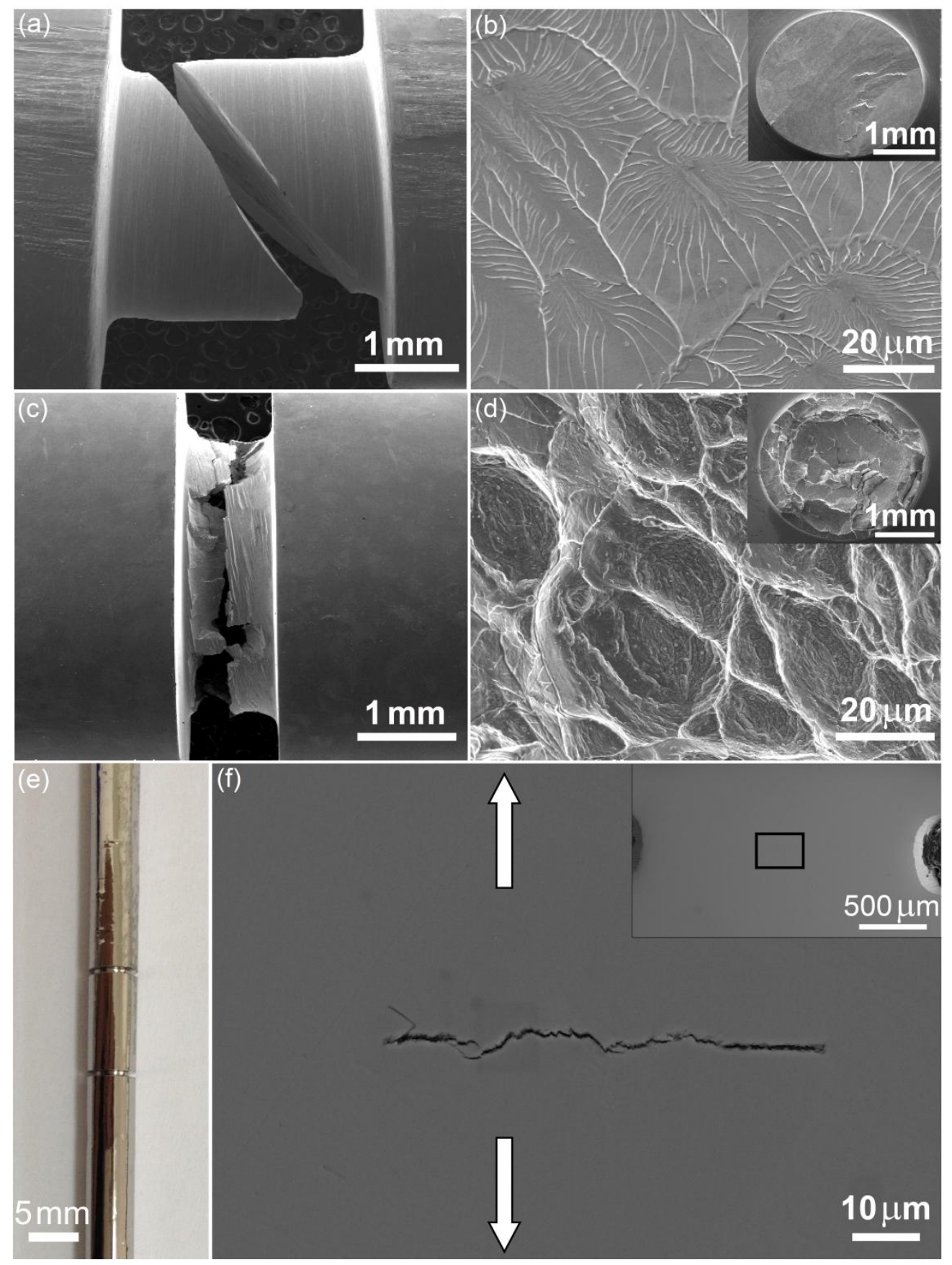


Figure 4

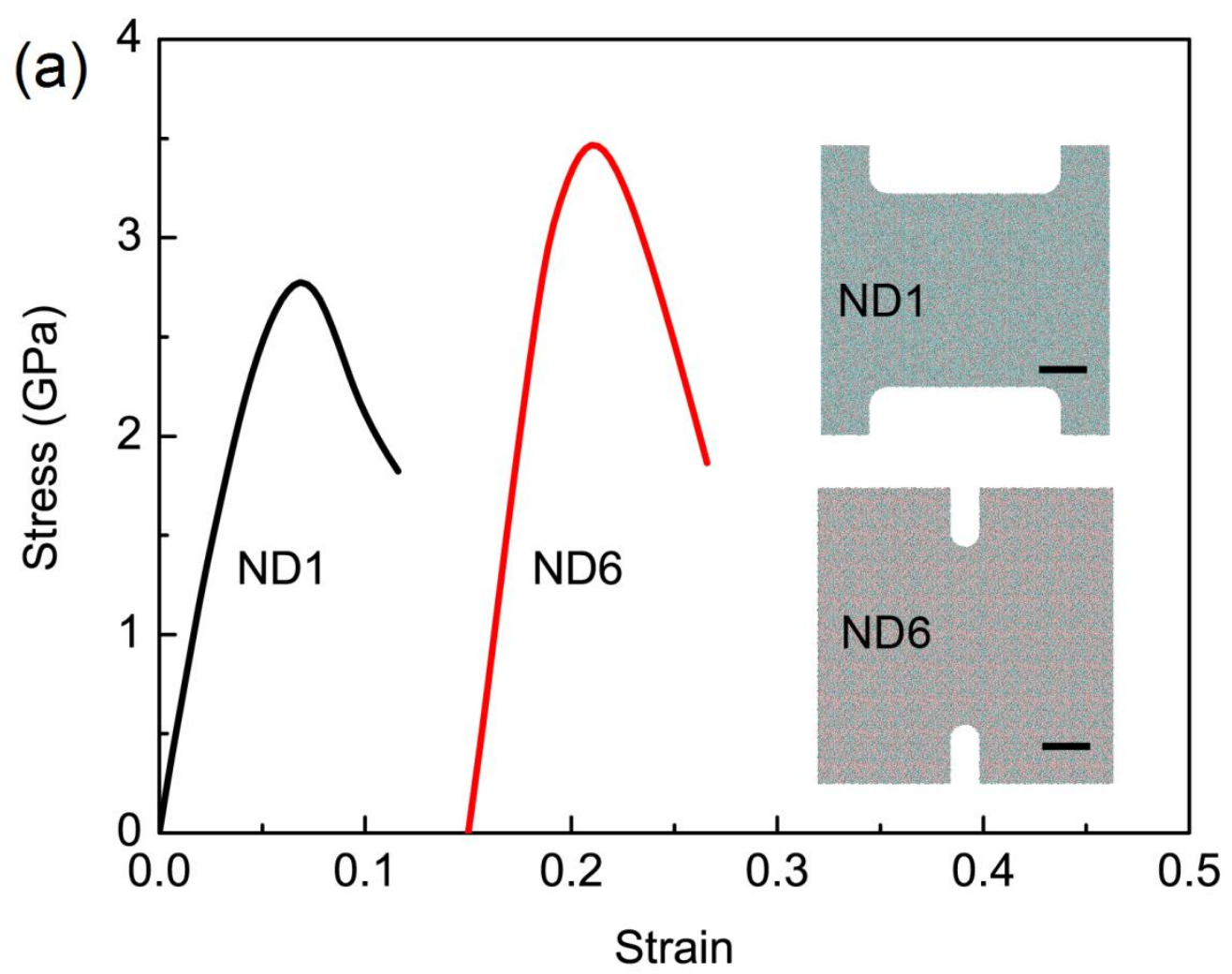

(b)

(c)
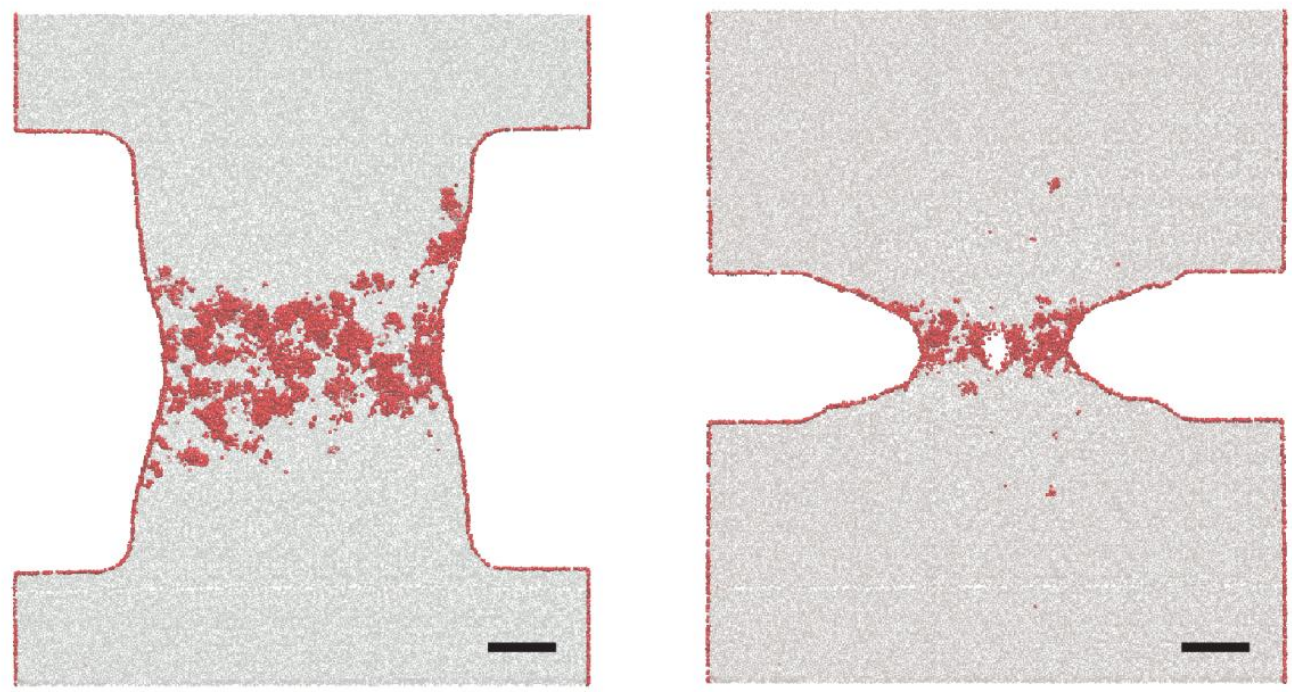


\section{Figure 5}

(a)

$\mathrm{MPa}$
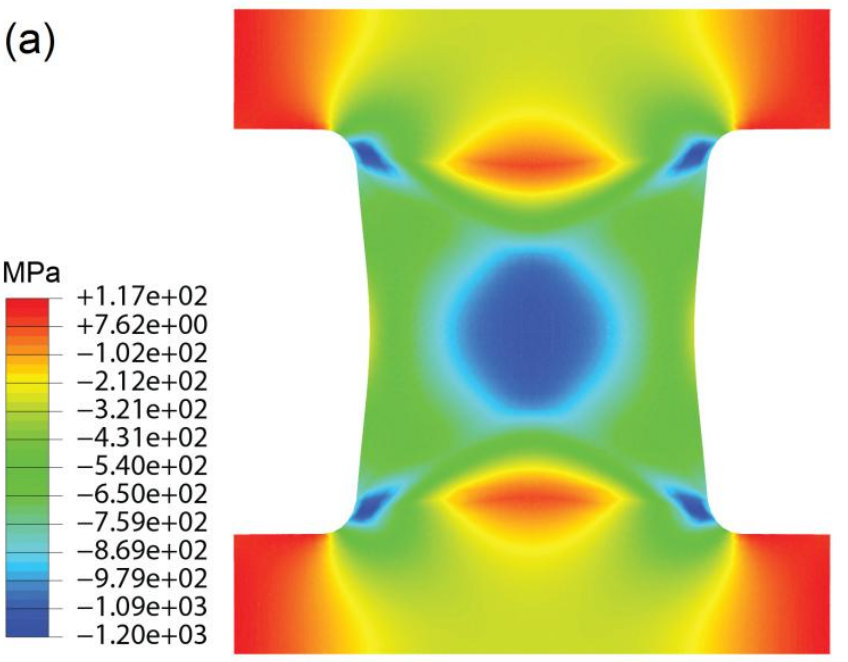

(b)

$\mathrm{MPa}$

$+4.96 \mathrm{e}+02$

$+1.82 \mathrm{e}+02$

$-1.32 \mathrm{e}+02$

$-4.45 e+02$

$-7.59 \mathrm{e}+02$

$-1.07 \mathrm{e}+03$

$-1.39 \mathrm{e}+03$

$-1.70 \mathrm{e}+03$

$-2.01 e+03$

$-2.33 e+03$

$-2.64 \mathrm{e}+03$

$-2.95 e+03$




Figure 6

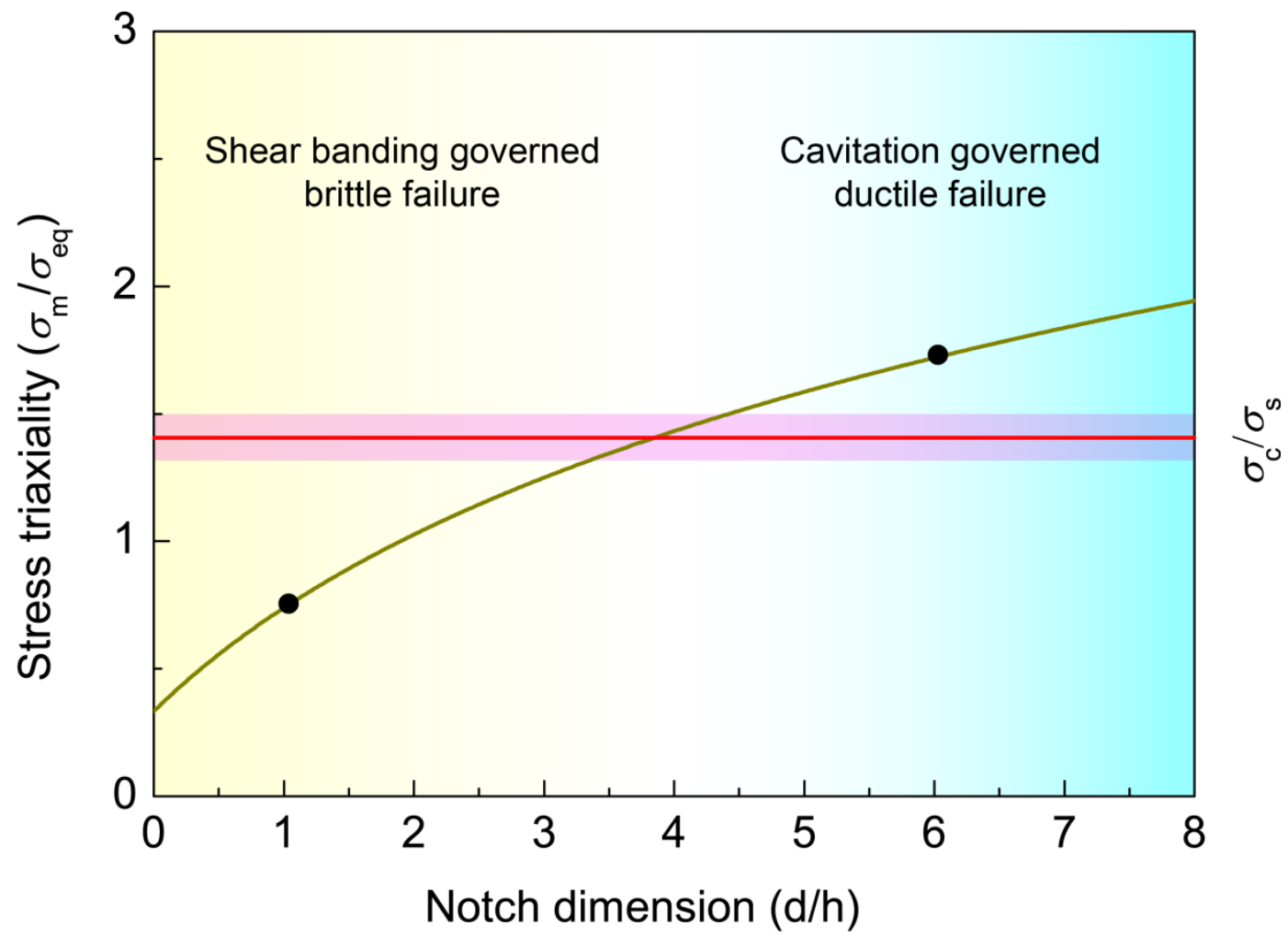


Figure 7



